\title{
Numerical simulation of compressible cavitating two-phase flows with a pressure-based solver
}

\author{
Marco Cristofaro ${ }^{1,2, *}$, Wilfried Edelbauer ${ }^{1}$, Manolis Gavaises ${ }^{2}$, Phoevos Koukouvinis ${ }^{2}$ \\ ${ }^{1}$ AVL List GmbH, Hans List Platz 1, 8020 Graz, AT \\ ${ }^{2}$ City University London, Northampton Square EC1V OHB, UK \\ ${ }^{*}$ Corresponding author: Marco.Cristofaro@avl.com
}

\begin{abstract}
This work intends to study the effect of compressibility on throttle flow simulations with a pressure-based solver. The simple micro throttle geometry allows easier access for obtaining experimental data compared to a real injector, but still maintaining the main flow features. For this reasons it represents a meaningful and well reported benchmark for validation of numerical methods developed for cavitating injector flows.

An implicit pressure-based compressible solver is used on the filtered Navier-Stokes equations. Thus, no stability limitation is applied on the time step. A common pressure field is computed for all phases, but different velocity fields are solved for each phase, following the multi-fluid approach. The liquid evaporation rate is evaluated with a Rayleigh-Plesset equation based cavitation model and the Coherent Structure Model is adopted as closure for the sub-grid scales in the momentum equation.

The aim of this study is to show the capabilities of the pressure-based solver to deal with both vapor and liquid phases considered compressible. A comparison between experimental results and compressible simulations is presented. Time-averaged vapor distribution and velocity profiles are reported and discussed. The distribution of pressure maxima on the surface and the results from a semi-empirical erosion model are in good agreement with the erosion locations observed in the experiments. This test case aims to represent a benchmark for further application of the methodology to industrial relevant cases.
\end{abstract}

\section{Keywords}

cavitation erosion, compressible pressure-based, multi-fluid LES

\section{Introduction}

Diesel injectors can suffer from cavitation erosion. Recent regulations in term of emissions forced engine manufacturers to increase the injection pressure to values above 2000 [bar]. The consequent higher velocities in the nozzle lead to the formation of a more diffused spray and, thus, better combustion. This causes higher engine efficiency and less emissions, but, at the same time, higher risk of cavitation. If the pressure reaches locally values below saturation pressure due to the high velocity, the liquid evaporates. Under certain conditions, the repetitive collapse of the generated vapor that is convected further downstream, where pressure is above the saturation pressure, may be an aggressive phenomenon. Fatigue damages can then appear on the nearby surfaces. Many works have been published in the last decades to predict cavitation erosion. Experimental campaigns are of crucial importance to gain a better understanding of the physical phenomena involved and to provide usable data for the validation of new models. Numerical approaches based on multi-phase Computational Fluid Dynamics (CFD) are able to model cavitating flow fields. Useful information can then be obtained to assess the probability of cavitation erosion appearance.

A wide range of flow aggressiveness and erosion risk indices were developed in the recent years. A review of some selected cavitation erosion models, together with a description of the physical mechanism leading to cavitation erosion, was provided by Van Terwisga et al. in [1]. The model of Bark et al. presented in [2, 3] is based on the analysis of collapsing vapor cavities dynamics and it well correlates the erosion risk with the underlying physical phenomena. On the other hand it may be difficult to use it when the erosive cavities are small. The model described in [4] by Fortes-Patella et al. is instead based on two efficiencies in which the energy is transferred from the collapsing cloud to the wall. The theoretical basis fit with the energy cascade phenomena and it could be easily applicable, but it still needs detailed studies to assess the efficiency values. In [5], Koukouvinis et al. defined a cavitation aggressiveness index based on the Lagrangian derivative of pressure and the time scales of a single bubble and of the whole vapor cavity. Bergeles et al. used instead the acoustic pressure of a single bubble collapse to develop an erosion aggressiveness index in [6]. The authors validated it on a real eroded injector geometry, that was also used by Koukouvinis et al. in [7] to test the cavitation aggressiveness index.

Multi-phase compressible CFD simulations were able to directly reproduce pressure waves generated by collapsing vapor clouds and impacting on the nearby surfaces. A 2-D inviscid density-based solver was used by Skoda et al. in [8] for a micro-channel flow and proved able to detect these pressure waves. In [9] Egerer et al. detected similar pressure peaks on the same geometry, but using a 3-D density-based solver with the single-fluid Large Eddy Simulation (LES) approach. A similar solver was also used by Mihatsch et al. in [10]. A grid dependency study of pressure waves intensity was performed and a scaling law was defined to fit the the pressure peaks rate to the one recorded during the experiments. Koukouvinis et al. presented in [11] the recorded pressure peaks obtained with a pressure-based solver with a single-fluid LES approach. Results are presented for a micro-channel flow, as 
well as for a real injector case. Another cavitating injector simulation was presented by Örley et al. in [12], where a density-based solver with explicit time integration and the single-fluid approach were applied. Pressure peak intensities in the order of 1000 [bar] were recorded in both works.

The present work exploits the pressure-based commercial solver AVL FIRETM resolving a fully compressible multiphase cavitating flow. Implicit time integration is adopted to avoid stability constraints on the simulation time step. The multi-fluid modeling approach avoids the need of mixture properties definition, instead required for the singlefluid approach. Both vapor and liquid compressibility are considered with separate equations of state. Furthermore the slip velocity between the phases is modeled and analyzed. Pressure peaks recorded on the surface are then presented and compared with the semi-empirical erosion model presented by Greif et al. in [13]. Experimental visualization of cavitation erosion is also included for comparison.

\section{Numerical model}

The set of Navier-Stokes equations describing the iso-thermal compressible 2-phase cavitating flow is hereby presented. The equations are iteratively solved with a pressure-based solver. In the applied methodology a single pressure is solved for all phases, but different velocity fields are obtained for each of them. This is known as Eulerian-Eulerian modeling approach, or multi-fluid model [14]. The dispersed phase (vapor) is then treated as a second continuous phase interpenetrating the continuous phase (liquid). The mass conservation equations are first solved independently to compute the volume fraction of each phase, $\alpha_{k}{ }^{*}$, and then combined to obtain the common pressure, $p$. Once the phase densities, $\rho_{k}$, are computed using appropriate equations of state (see Sec. Geometrical model and simulation set-up), two separate momentum conservation equations are solved to find the phase velocity fields, $\bar{v}_{k}$. The interaction between phases must be included in the mass and momentum exchange terms. In the present methodology they are modeled considering a mono-dispersed bubbly flow model [15].

The mass conservation of each phase is represented by a transport equation with a source term modeling the inter-phase mass transfer, $\Gamma_{k}$. Since the total mass must be conserved, the mass lost by one phase is absorbed by the other and then: $\sum_{k} \Gamma_{k}=0$. Equation 1 shows the mass conservation equation in the differential conservative formulation for the generic phase $k$.

$$
\frac{\partial \alpha_{k} \rho_{k}}{\partial t}+\nabla \cdot \alpha_{k} \rho_{k} \bar{v}_{k}=\Gamma_{k}
$$

Equation 1 is used to compute the volume fraction of both the liquid and the vapor phase, assuming densities and velocities as known. Since the values of volume fraction do not satisfy a priori the condition of exactly filling the available volume, a compatibility condition is also imposed $\sum_{k} \alpha_{k}=1$.

The inter-phase mass transfer is modeled with the Original FIRE linear cavitation model [16]. The model considers the mass transfer contribution from each vapor bubble dynamic, as presented in Eq. 2.

$$
\Gamma_{d}=-\Gamma_{c}=N \rho_{d} 4 \pi R^{2} \dot{R}=\rho_{d}\left(3 \alpha_{d}\right)^{2 / 3}(4 \pi N)^{1 / 3} \dot{R}
$$

The vapor bubble number density is indicated with $N$, the average bubble radius with $R$ and its rate of change $\dot{R}$. Positive values of $\Gamma_{d}$ mean a growth of the bubble size, thus evaporation of the liquid into vapor. The bubble radius rate of change is extracted from the simplified Rayleigh-Plesset equation for a single bubble [17, 18]. No thermal, gas content, viscosity and surface tension effects are considered in the equation, and the second order time derivative term is neglected. The final formulation for $\dot{R}$ is then given in Eq. 3 .

$$
\dot{R}= \pm \sqrt{\frac{2}{3} \frac{\left|p_{\text {sat }}-p\right|}{\rho_{c}}} \text { with } \begin{cases}+ & \text { if } p \leq p_{\text {sat }} \\ - & \text { if } p>p_{\text {sat }}\end{cases}
$$

The saturation pressure of the liquid is indicated with $p_{\text {sat }}$. The number density is instead calculated according to an empirical function, formed by a constant part and a diminishing linear ramp, see Eq. 4 .

$$
N= \begin{cases}N_{0} & \text { if } \alpha_{d} \leq 0.5 \\ \max \left[2\left(N_{0}-1\right)\left(1-\alpha_{d}\right)+1, N_{\text {min }}\right] & \text { if } \alpha_{d}>0.5\end{cases}
$$

This is a rather heuristic model used to model coalescence effects: as soon as the vapor volume fraction is greater than 0.5 the bubbles start to merge. The initial number density, $N_{0}$, strongly depends on the characteristics of the liquid phase and it is the main cavitation model parameter. A minimum value, $N_{m i n}$, is also imposed.

$A$ joined mass conservation equation is then obtained by combining all the mass transport equations. Considering $\alpha_{k}$ and $\bar{v}_{k}$ as known, Eq. 5 is solved to compute the pressure shared among all phases. It is important to notice that the term $\Gamma_{k}$ is not elided but it is a volumetric flux when phase change occurs.

$$
\sum_{k} \frac{1}{\rho_{k}}\left\{\frac{\partial \alpha_{k} \rho_{k}}{\partial t}+\nabla \cdot \alpha_{k} \rho_{k} \bar{v}_{k}-\Gamma_{k}\right\}=0
$$

\footnotetext{
* The subscript $k$ is used to indicate a quantity related to a generic phase. The letter $c$ is instead used for the continuous liquid phase and $d$ for the dispersed vapor phase.
} 
The density time derivative of the first term of Eq. 5 is elaborated considering the compressibility in Eq. 6 . The isentropic phase speed of sound is defined as $c_{k}^{2}=\left(\partial p / \partial \rho_{k}\right)_{s}$. The final formulation shows how the presented pressure equation is in agreement with Wood's mixture speed of sound $1 / \rho_{m} c_{m}^{2}=\sum_{k} \alpha_{k} / \rho_{k} c_{k}^{2}$, [19] (being $\rho_{m}=\sum_{k} \alpha_{k} \rho_{k}$ ).

$$
\sum_{k} \frac{\alpha_{k}}{\rho_{k}} \frac{\partial \rho_{k}}{\partial t}=\sum_{k} \frac{\alpha_{k}}{\rho_{k}} \frac{\partial \rho_{k}}{\partial p} \frac{\partial p}{\partial t}=\left\{\sum_{k} \frac{\alpha_{k}}{\rho_{k}} \frac{1}{c_{k}^{2}}\right\} \frac{\partial p}{\partial t}=\frac{1}{\rho_{m} c_{m}^{2}} \frac{\partial p}{\partial t}
$$

One momentum conservation equation is then solved for each phase separately as presented in Eq. 7 . The momentum transfer and turbulence models provide the closure terms.

$$
\frac{\partial \alpha_{k} \rho_{k} \bar{v}_{k}}{\partial t}+\nabla \cdot \alpha_{k} \rho_{k} \bar{v}_{k} \bar{v}_{k}^{T}=-\alpha_{k} \nabla p+\nabla \cdot \alpha_{k}\left(\boldsymbol{T}_{k}+\boldsymbol{T}_{k}^{s g s}\right)+\bar{M}_{k}+\bar{v}_{i} \Gamma_{k}
$$

The vector $\bar{M}_{k}$ is the inter-phase momentum transfer and $\bar{v}_{i}$ is the interface velocity. The superscript $T$ indicates the transpose of a vector or a matrix, so that $\bar{v}_{k}^{T}$ is an horizontal vector, being $\bar{v}_{k}$ considered vertical. The term $\bar{v}_{i} \Gamma_{k}$ represents the momentum carried by the mass transferred between phases. The $3 \times 3$ matrix $\boldsymbol{T}_{k}$ is the shear stress tensor, while $\boldsymbol{T}_{k}^{\text {sgs }}$ encloses the effect of the turbulence on the momentum conservation equation. In the present work the Large Eddy Simulation Coherent Structure Model (LES-CSM) is adopted as closure model [20].

The interfacial momentum exchange is modeled with the drag force acting on each bubble. The bubbles move into the liquid with a certain relative velocity: $\bar{v}_{r}=\bar{v}_{d}-\bar{v}_{c}$. This relative velocity causes a pressure distribution on the bubble surface, whose integral is a force opponent to the relative motion. Equation 8 presents the sum of drag forces in the mono-dispersed case.

$$
\left(\bar{M}_{c}\right)_{D}=N 4 \pi R^{2} \frac{1}{2} \rho_{c}\left|\bar{v}_{r}\right| \bar{v}_{r} C_{D}=(36 \pi N)^{1 / 3} \alpha_{d}^{2 / 3} \frac{1}{2} \rho_{c}\left|\bar{v}_{r}\right| \bar{v}_{r} C_{D}
$$

The number density is taken consistently from the cavitation model, see Eq. 4, and the drag coefficient, $C_{D}$, is modeled as presented in [15, 21].

The presented equations are solved on the 3-D domain with the finite volume discretization method and the convergence of the system of equations is obtained with a Semi-Implicit Method for Pressure-Linked Equations (SIMPLE) algorithm, for further details see [15]. One benefit of pressure-based solvers is that the same numerical procedure can be used for both compressible and incompressible simulations. Originally pressure-based solvers were developed to solve incompressible flow fields. Thanks to further developments, their applicability was extended also to compressible flows. Consequently, a direct comparison between compressible and incompressible flow fields simulations can be obtained, with no major modifications in the numerical algorithm. The opportunity to use wellconsolidated solution procedure, along with existing cavitation models, makes pressure-based solvers of great interest for both scientific and industrial communities.

\section{Experimental setup}

The experimental data used as reference in this work are already published in [22]. The setup was designed to study cavitation erosion for a micro-throttle flow, representing a simplified Diesel injector nozzle. A sharp-edge geometry is considered, also known as I-channel geometry. The main components of the hydraulic system were a tank, an high pressure pump, a metal throttle and a cooling system. The throttle is enclosed between two sapphire glasses that allow optical access to the flow. The channel is $993[\mu \mathrm{m}]$ long, $295[\mu \mathrm{m}]$ high and $300[\mu \mathrm{m}]$ depth. The working fluid was commercially available Diesel fuel and it was demonstrated that small variations of the properties (e.g. due to a different manufacturer) did not influence the results [22]. Figure 1 shows an exploded view of the test chamber with the flow path (left), the steel plate with the throttle (middle) and a schematic visualization of the throttle with dimensions in microns (right). An extensive description of the hydraulic system, fluid dynamic field, measurements techniques and data elaboration can be found in [8, 23, 22, 24, 25, 26].

In the present work the case with an inlet pressure of 300 [bar] and outlet pressure of 120 [bar] is considered. At this condition the pressure waves intensity induced by collapsing vapor clouds are maximum [8]. This working condition is also known as cavitation critical point (CCP) [22]. It separates the states for which by increasing the pressure difference between inlet and outlet, the mass flow increases (following the Bernoulli curve) or remains constant (choked flow). The CCP can change with inlet and outlet pressure, temperature and fluid quality (e.g. nuclei presence and dissolved gas).

\section{Geometrical model and simulation set-up}

Since cavitation is an inertial driven phenomenon, thermal effects are here neglected, assuming an iso-thermal flow field. The fluid properties of Diesel described in [22] have been used. The liquid continuous phase density is modeled with a linearized equation of state as described in Eq. 9.

$$
\rho_{c}(p)=\rho_{r e f}+\left(\frac{\partial \rho}{\partial p}\right)_{r e f}\left(p-p_{r e f}\right)=\rho_{r e f}+\frac{p-p_{r e f}}{c^{2}}
$$



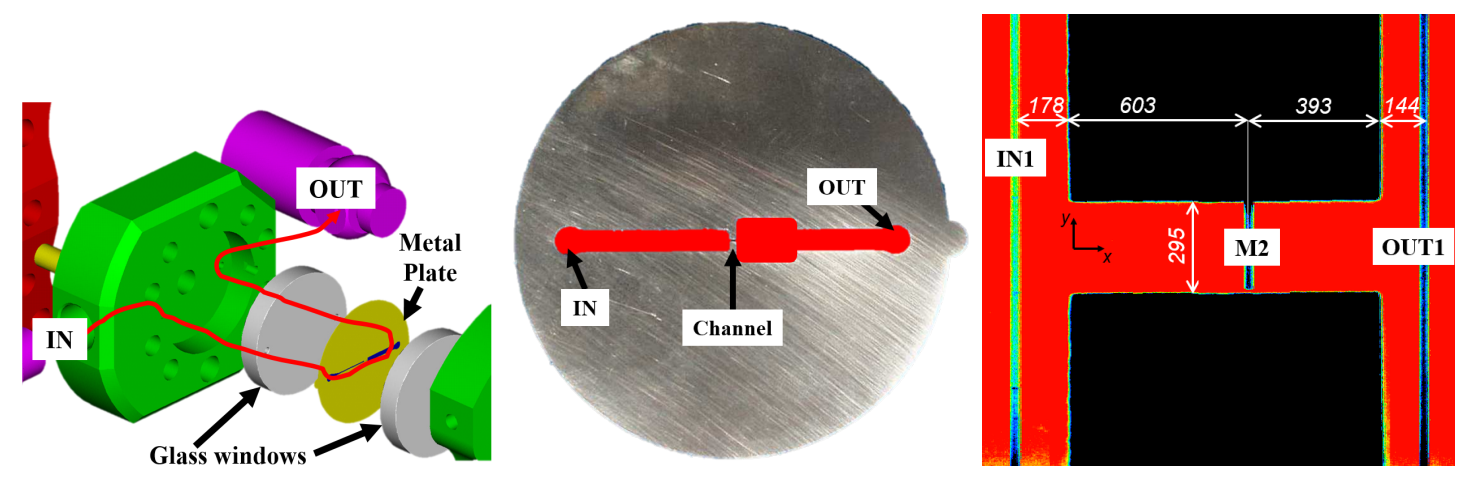

Figure 1. Micro throttle of the Prevero test case: flow path (left), steel plate (middle) and geometry with dimensions in microns of the throttle (right). Reproduced from [22]

The reference density, $\rho_{\text {ref }}$, was set to $820\left[\mathrm{~kg} / \mathrm{m}^{3}\right]$, reference pressure, $p_{\text {ref }}$, to 1 [bar] and the iso-thermal liquid speed of sound, $c$, to $1320[\mathrm{~m} / \mathrm{s}]$. The saturation pressure, $p_{\text {sat }}$, was of $4500[\mathrm{~Pa}]$ and a dynamic viscosity of 0.0021 [Pas] was used accordingly to [23]. The Diesel vapor density was instead modeled with the ideal gas law, see Eq. 10.

$$
\rho_{d}(p)=\frac{p}{R_{g} T}
$$

The specific gas constant, $R_{g}$, was of $48[\mathrm{~J} / \mathrm{kg} \mathrm{K}]$ and a constant temperature, $T$, of $321.15[\mathrm{~K}]$ was used. The vapor phase dynamic viscosity was set to $10^{-5}$ [Pas].

The used computational mesh was presented in [23] for the LES simulations. It is a block structured volume mesh and it is composed of 17 million cells. The smallest cell dimension is of $8.210^{-7}$ [m] close to the wall, while the characteristic cell size inside the channel is of $2.910^{-6}[\mathrm{~m}]$. The characteristic length scales of the liquid flow can be estimated from preliminary Reynolds-Averaged Navier-Stokes (RANS) simulations. The Kolmogorov length scale is computed as $\eta=\left(\nu^{3} / \mathcal{E}\right)^{1 / 4} \simeq 210^{-7}[\mathrm{~m}]$ and the Taylor length scale is estimated as $\lambda=\sqrt{10} \operatorname{Re}^{-1 / 2} L \simeq 510^{-6}[\mathrm{~m}]$. The used values are: turbulence dissipation rate $\mathcal{E} \simeq 10^{10}\left[\mathrm{~m}^{2} / \mathrm{s}^{3}\right]$, kinematic viscosity $\nu \simeq 2.510^{-6}\left[\mathrm{~m}^{2} / \mathrm{s}\right]$, velocity $|\bar{v}|=270[\mathrm{~m} / \mathrm{s}]$ and length $L=310^{-4}[\mathrm{~m}]$. The characteristic cell size inside the channel is then circa fifteen times the Kolmogorov length scale and half of the Taylor length scale. From the preliminary RANS results it is possible to compute the first cell $y^{+}$value as $y^{+}=y(1) / \ell_{\tau} \simeq 1.4$ (being $\ell_{\tau} \simeq 510^{-7}[\mathrm{~m}]$ ).

Static pressure was imposed at the open boundaries: 300 [bar] was applied at the inlet and 120 [bar] at the outlet. The central differencing scheme was applied to the momentum equation, while the MINMOD scheme was used for pressure and volume fraction equations, [27]. A small blending with the upwind scheme was added to the momentum equation to damp spurious numerical oscillations. A constant time step of $10^{-8}[\mathrm{~s}]$ was adopted, corresponding to a maximum value of convective CFL number of 3 . The time marching is done with an unconditionally stable second order accurate three time level implicit scheme. Although the maximum value of the acoustic CFL number based on the liquid speed of sound is 16 , the compressibility of the mixture corresponds to a speed of sound lower than the one of the pure liquid phase [19], leading to acoustic CFL numbers in liquid-vapor mixture regions smaller than one. The following numerical procedure was adopted to decrease the computational effort of the simulation. Firstly a RANS simulation was run for $210^{-4}$ [s] on a coarse grid with no mass transfer allowed from vapor to liquid. A quasi-steady solution was then obtained, with pure liquid in the whole domain. The obtained flow field was used to initialize the LES on the finer grid. The simulation run then for further $510^{-5}$ [s] for the evaluation of the results. This allowed to strongly reduce the overall computational cost compared to start the simulation directly with LES on the fine mesh.

\section{Results and discussion}

Results from the compressible numerical simulation are presented in this section. Both time-averaged and instantaneous values are included in the analysis. The integral mass flow and the vapor cavity distribution from simulations are compared with the experimental data reported in [22]. Mid-cut flow fields are then presented, together with the corresponding velocity profiles. Two cavitation erosion prediction approaches are finally compared with the experiments.

Table 1 presents the time-averaged mass flow values obtained in the experiment and in the simulation. For the simulation, a time average of the integral mass flow over the cross section M2 (see Fig. 1) was computed. The simulation shows a $4.25 \%$ smaller average mass flow compared to the experiments. The reason for this discrepancy is attributed to the different vapor distribution obtained from the simulation compared to the experiment. A bigger area occupied by the vapor in the channel cross section leads to sligthly smaller mass flow rate values. A further reason for the difference is the very small averaging time obtained from the simulation compared to the experiments, due to the high computational cost. However, considering the standard deviation of the simulation, the experiment result lays in the statistical range of the values from the simulation. 
Table 1. Average mass flow comparison. Experimental value from [22] against the simulation average integral mass flow at $M 2$ with the corresponding standard deviation.

\begin{tabular}{ccc}
\hline Experimental & Simulation & Relative difference \\
\hline $12.7[\mathrm{~g} / \mathrm{s}]$ & $12.16 \pm 0.55[\mathrm{~g} / \mathrm{s}]$ & $-4.25 \%$
\end{tabular}

In Fig. 2 the vapor distribution obtained from 50 transmission images (left) is compared with the maximum value of time-averaged vapor volume fraction along the channel depth (right). The flow moves from the left to the right. The maximum value along the $z$ direction (being $z$ the coordinate starting from the bottom glass window, perpendicular to it and orientated into the channel) was adopted to mimic the physics of the experiments, for which the light is blocked by the presence of vapor at any location along its path.

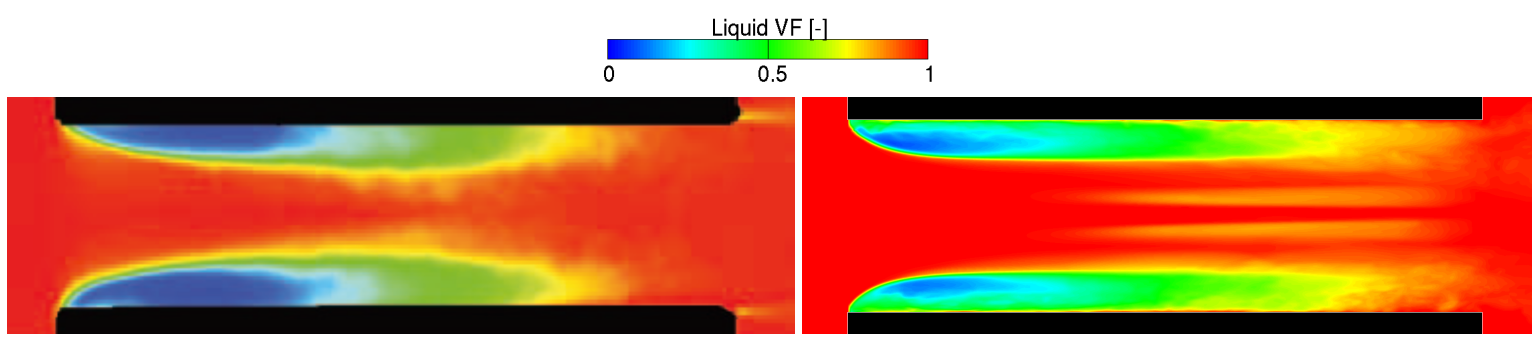

Figure 2. Time-averaged vapor cavity distribution (red liquid, blue vapor). Experimental transmission images reproduced from [23] (left) and minimum value along depth from simulation results (right).

The average flow field obtained from the simulation is further described in Fig. 3. A cut at the half of the domain depth is presented for the liquid velocity and the average pressure fluctuations fields. A vapor cavity along the entire channel length close to the walls can be observed in both the vapor (Fig. 2) and the velocity field. The cavity closes at the right end of the channel, where the highest values of time-averaged pressure fluctuations appear.

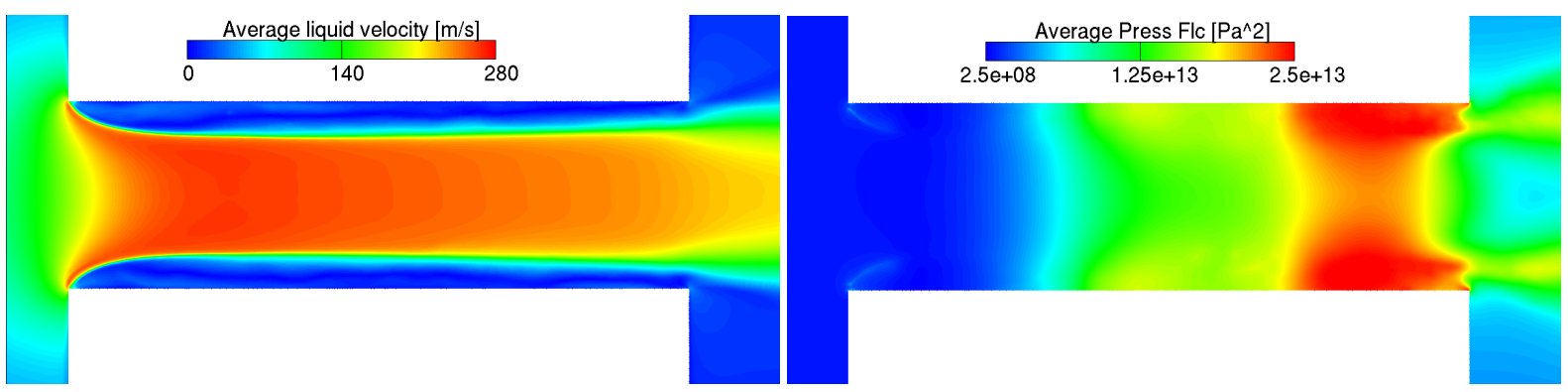

Figure 3. Time-averaged liquid velocity magnitude (left) and pressure fluctuations (right). Mid-cut of simulation results.

Figure 4 shows a sample of an instantaneous velocity and a volume fraction field. The laminar to turbulent transition of the shear layer can be detected in the velocity field at around half of the channel length. The dynamics of the vapor phase is instead shown in the volume fraction field: unstable vapor clouds detach from the main cavity and are convected downstream. They are then subjected to further break-up, collapse and rebounding. A strong dynamic interaction is detected between collapsing vapor clouds and pressure waves. This is recorded by the strong impact pressure on the nearby surfaces, usually related with possible cavitation erosion, presented later in this work.

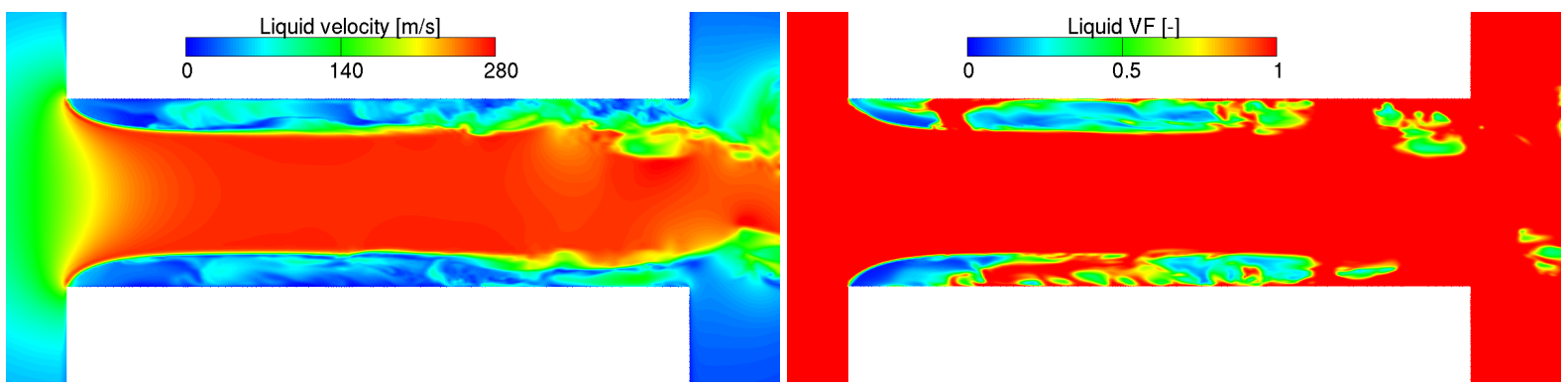

Figure 4. Instantaneous velocity magnitude (left) and liquid volume fraction (right). Mid-cut of simulation results at $t=3.510^{-5}[\mathrm{~s}]$. 
The numerical model used in the current work is based on the solution of two separate momentum equations. This leads to the computation of a separate velocity field for each phase. The slip velocity can then be evaluated as the difference between the two velocity fields. Figure 5 presents an example of instantaneous liquid velocity and relative slip velocity (defined as the velocity difference non-dimensionalised with the liquid velocity). The values are taken from three measurements planes IN1, M2 and OUT1 according to Fig. 1 (right) and then extracted at half of the channel depth $(z=150[\mu \mathrm{m}])$. The three different locations are presented on the same graphs. Slip velocities of the order of $15 \%$ of the liquid velocity were detected. It is important to notice that the highest values appear for M2 and OUT1 sections in liquid-vapor mixture regions.
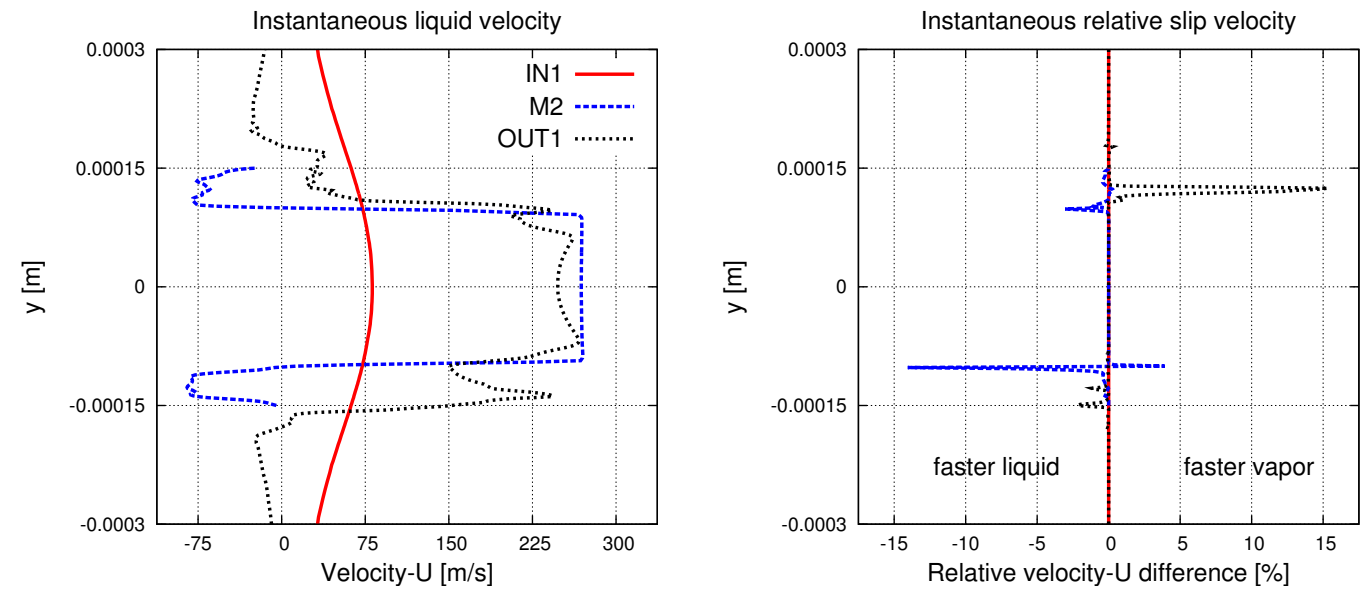

Figure 5. Instantaneous liquid velocity profiles (left) and relative slip velocity (right). Local values from a mid-cut of the simulation results at $t=3.510^{-5}[\mathrm{~s}]$.

In Fig. 6 the same analyses as in Fig. 5 is presented but for time-averaged velocities. The maximum magnitude of the average slip velocity is of the order of $1.5 \%$ of the liquid velocity. This is 10 times lower than the instantaneous value, but it still appears in the liquid-vapor mixture regions along M2 and OUT1 sections.
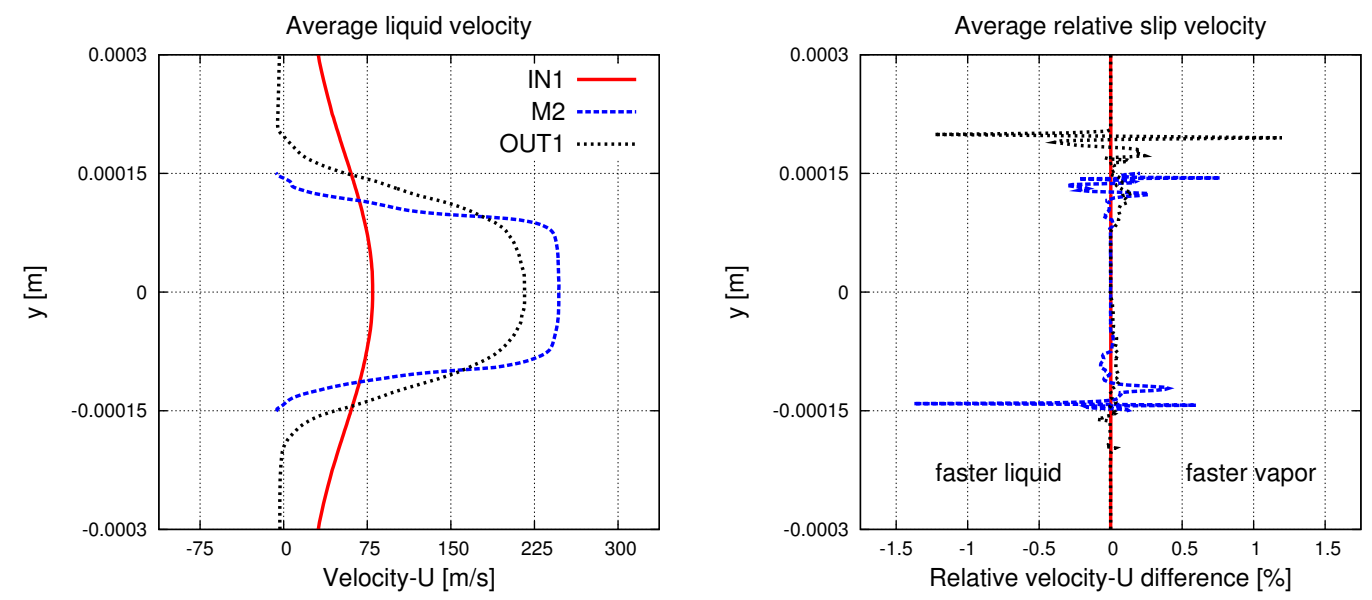

Figure 6. Average liquid velocity profiles (left) and relative slip velocity (right). Local values from a mid-cut of the simulation results.

The results of two different cavitation erosion modeling approaches are then presented and compared with experiments. The cavitation erosion prediction model described in [15] and already applied in [13, 23], is compared with the pressure peaks recorded on the channel surface during the simulation. The compressibility of both liquid and vapor phases was essential to resolve pressure waves starting from collapsing vapor clouds.

The maximum recorded pressure on the surface was 1500 [bar], however it is important to mention that this value is mesh and time step dependent [10]. In the present work the distribution of recorded pressure peaks is only used to obtain a qualitatively distribution of possible erosion locations. Figure 7 presents the qualitative results of erosion risk prediction from the modeling of the Mean Depth of Penetration Rate (MDPR) (left above) and the recorded pressure peaks (right above), together with experimental visualization of the erosion time evolution from [8] (below). A black cut at half of the depth of the channel is included for the simulation results, showing the average vapor presence in gray-scale. The same geometry was used for both the experiment and the simulation. The experimental data were obtained with 125 [bar] at the outlet and a temperature of $60\left[{ }^{\circ} \mathrm{C}\right]$. The identical operational point was not simulated due to the lack of flow experimental analyses for this exact condition. The red arrows show 
an area that both MDPR model and pressure peaks detect as regions with high cavitation erosion risk. A similar cavitation erosion location was also found in the experiments. This demonstrates the capability to detect correct cavitation erosion locations with both modeling approaches. The evolution of experimental erosion patterns after the first material removal cannot be directly compared with simulations due to the modifications in the geometry.
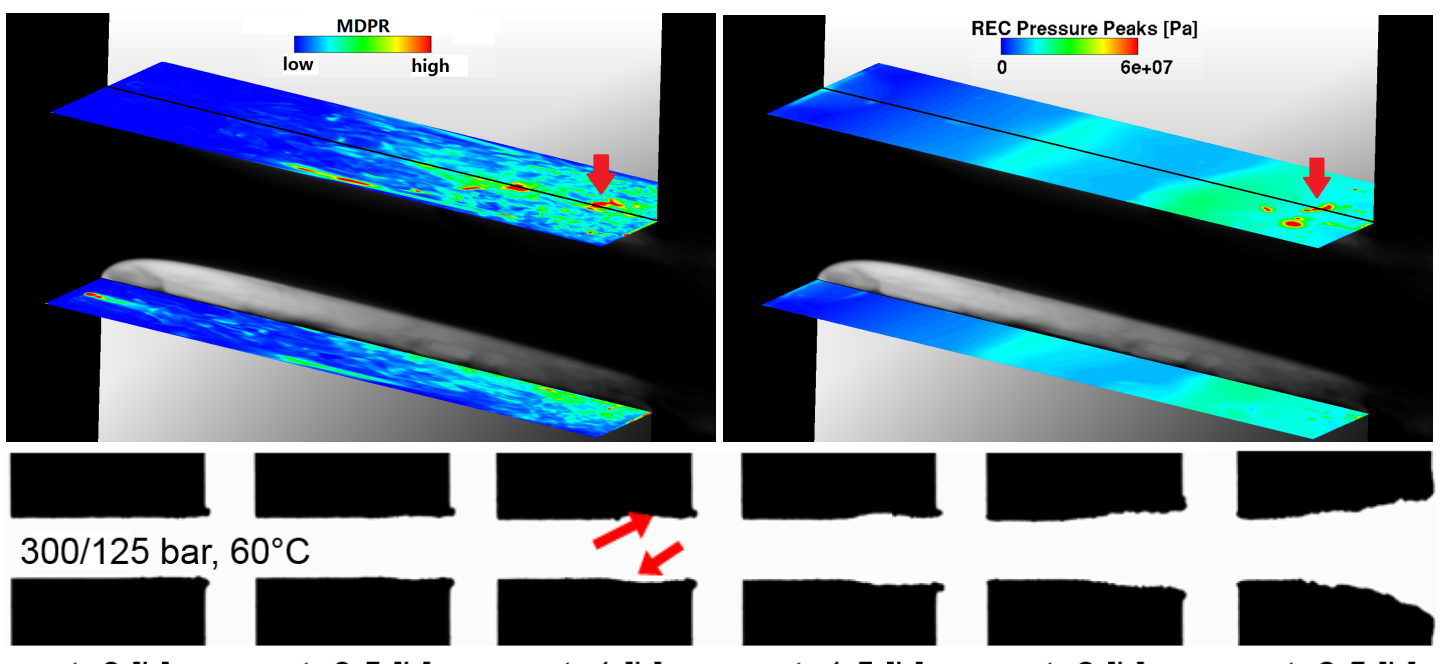

$t=1.5[h]$

$\mathrm{t}=2[\mathrm{~h}]$

$\mathrm{t}=2.5[\mathrm{~h}]$

Figure 7. Cavitation erosion risk predictions comparison. Averaged modeled MDPR (left), maximum recorded pressure peaks (right) and experimental cavitation erosion time evolution reproduced from [8] (below).

\section{Conclusions}

A numerical approach to solve compressible multi-phase flow fields is presented and results for a micro-throttle cavitating flow are discussed. A pressure-based solver is used to resolve iso-thermal multi-phase flow fields, with all phases considered compressible. Separate velocity fields are also computed for each phase. The computational methodology is presented, including mass and momentum transfer models for cavitating flows. The capability of the pressure-based solver to resolve compressible multi-phase cavitating flows is then assessed.

The I-channel test case is then used for verification. Average mass flow and vapor distribution are showed in good agreement with experimental data. The average and instantaneous flow fields resulting from the simulation are then illustrated, including an analysis of the two velocity profiles differences. It is demonstrated that the slip velocity modeled with the multi-fluid approach is significant for local and instantaneous values, but it is reduced on time-averaged velocity fields.

The capabilities of different cavitation erosion prediction models are finally analyzed, with reference to experimental visualizations. Surface pressure peaks above the driving pressure could be detected with the presented compressible solver. A good agreement exists between the erosion locations of the semi-empirical erosion model and the recorded surface pressure peaks with the experimental erosion patterns.

\section{Acknowledgements}

Financial support from the MSCA-ITN-ETN of the European Union's H2020 programme, under REA grant agreement n. 642536 is acknowledged.

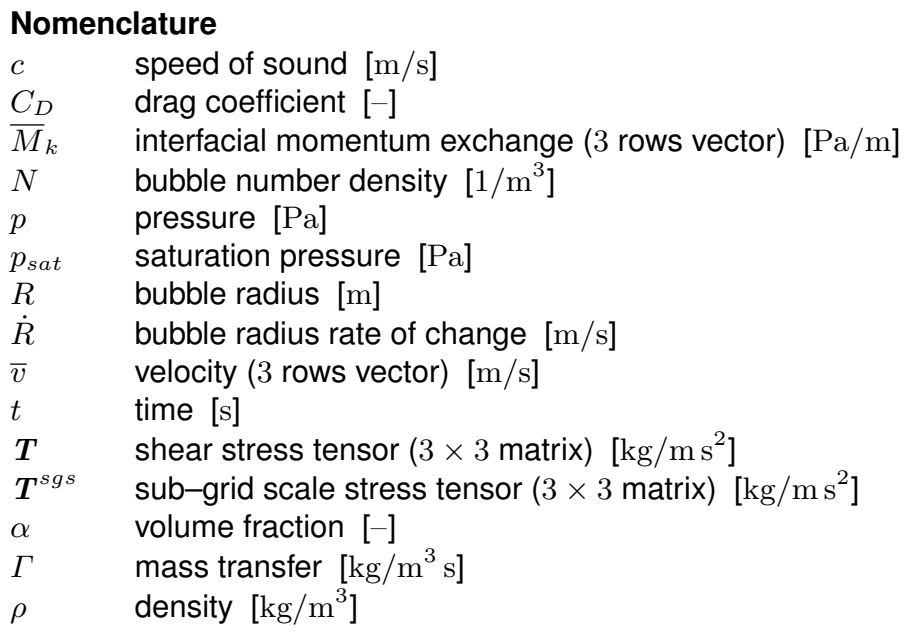




\section{References}

[1] Van Terwisga, T. J. C., Fitzsimmons, P. A., Ziru, L., and Foeth, E. J., 2009. "Cavitation erosion - a review of physical mechanisms and erosion risk models". In 7th International Symposium on Cavitation.

[2] Bark, G., Berchiche, N., and Grekula, M., 2004. Application of principles for observation and analysis of eroding cavitation - the EROCAV observation handbook. Edition 3.1.

[3] Grekula, M., and Bark, G., 2009. "Analysis of video data for assessment of the risk of cavitation erosion". In 1st International Conference on Advanced Model Measurement Technology for the EU Maritime Industry.

[4] Fortes-Patella, R., Reboud, J. L., and Briancon-Marjollet, L., 2006. "A phenomenological and numerical model for scaling the flow agressiveness in cavitation erosion". In EROCAV Workshop.

[5] Koukouvinis, P., Bergeles, G., and Gavaises, M., 2015. "A cavitation aggressiveness index within the Reynolds averaged Navier Stokes methodology for cavitating flows". Journal of Hydrodynamics, 27(4), pp. 579-586. http://dx.doi.org/10.1016/S1001-6058(15)60519-4.

[6] Bergeles, G., Li, J., Wang, L., Koukouvinis, F., and Gavaises, M., 2015. "An erosion aggressiveness index (EAI) based on pressure load estimation due to bubble collapse in cavitating flows within the RANS solvers". SAE Int. J. Engines, 8(5). http://dx.doi.org/10.4271/2015-24-2465.

[7] Koukouvinis, P., Bergeles, G., Li, J. Z., Wang, L., Theodorakakos, A., and Gavaises, M., 2015. "Simulation of cavitation inside diesel injectors, including erosion modelling". In Fuel Systems for IC Engines, IMECHE, pp. 125-135.

[8] Skoda, R., Iben, U., Mozorov, A., Mihatsch, M., Schmidt, S. J., and Adams, N. A., 2011. "Numerical simulation of collapse induced shock dynamics for the prediction of the geometry, pressure and temperature impact on the cavitation erosion in micro channels". In WIMRC, 3rd International Cavitation Forum.

[9] Egerer, C. P., Hickel, S., Schmidt, S. J., and Adams, N. A., 2014. "Large-eddy simulation of turbulent cavitating flow in a micro channel”. Physics of Fluids, 26(085102), pp. 190-200. http://dx.doi.org/10.1063/1.4891325.

[10] Mihatsch, M. S., Schmidt, S. J., and Adams, N. A., 2015. "Cavitation erosion prediction based on analysis of flow dynamics and impact load spectra". Physics of Fluids, 27(103302). http://dx.doi.org/10.1063/1.4932175.

[11] Koukouvinis, P., Gavaises, M., Li, J. Z., and Wang, L., 2016. "Large eddy simulation of diesel injector including cavitation effects and correlation to erosion damage". Fuel, 175, February, pp. 26-39. http://dx.doi.org/10.1016/j.fuel.2016.02.037.

[12] Örley, F., Hickel, S., Schmidt, S. J., and Adams, N. A., 2016. "Large-eddy simulation of turbulent, cavitating fuel flow inside a 9-hole diesel injector including needle movement". International Journal of Engine Research, April, p. 1468087416643901. https://doi.org/10.1177/1468087416643901.

[13] Greif, D., and Wang, D. M., 2006. "Aspects of modeling cavitation effects within injection equipment using advanced two-fluid techniques". Turbulence, Heat and Mass Transfer, 5, pp. 769-772. http://dx.doi.org/10.1615/ICHMT.2006.TurbulHeatMassTransf.1680.

[14] Brennen, C. E., 2005. Fundamentals of Multiphase Flows. Cambridge University Press. ISBN 0521848040.

[15] AVL FIRE TM , 2017. CFD User Manual, v2017 ed.

[16] Grogger, H. A., and Alajbegović, A., 1998. "Calculation of the cavitating flow in Venturi geometries using two fluid mode". In ASME Fluids Engineering Division Summer Meeting, A. FEDSM, ed.

[17] Lord Rayleigh, J. W. S., 1917. "On the pressure developed in a liquid during the collapse of a spherical cavity". Philosophical Magazine Series 6, 34(200), pp. 94-98. http://dx.doi.org/10.1080/14786440808635681.

[18] Plesset, M. S., 1949. "The dynamics of cavitation bubbles". ANSME Journal of Applied Mechanics, 16, pp. 228-231.

[19] Wood, A. B., 1930. A textbook of sound: Being an account of the physics of vibrations with special reference to recent theoretical and technical developments. Macmillan.

[20] Kobayashi, H., 2005. "The subgrid-scale models based on coherent structures for rotating homogeneous turbulence and turbulent channel flow". Physics of Fluids, 17(4), p. 045104.

[21] Ishii, M., and Mishima, K., 1984. "Two-fluid model and hydrodynamic constitutive relations". Nuclear Engineering \& Design, 8(107).

[22] Morozov, A., and Iben, U., 2008. "Experimental analysis and simulation of cavitating throttle flow". In HEFAT, 6th International Conference on Heat Transfer, Fluid Mechanics and Thermodynamics.

[23] Edelbauer, W., Strucl, J., and Morozov, A., 2014. "Large eddy simulation of cavitating throttle flow". In SimHydro: Modelling of rapid transitory flows.

[24] Winklhofer, E., Kull, E., Kelz, E., and Morozov, A., 2001. "Comprehensive hydraulic and flow field documentation in model throttle experiments under cavitation conditions". In ILASS-Europe.

[25] Iben, U., Mozorov, A., Winklhofer, E., and Skoda, R., 2011. "Optical investigations of cavitating flow phenomena in micro channels using a nano second resolution". In WIMRC, 3rd International Cavitation Forum.

[26] Iben, U., Mozorov, A., Winklhofer, E., and Wolf, F., 2011. "Laser-pulse interferometry applied to high-pressure fluid flow in micro channels". Exp Fluids(50), pp. 597-611. http://dx.doi.org/10.1007/s00348-010-0950-9.

[27] Sweby, P. K., 1984. "High resolution schemes using flux limiters for hyperbolic conservation laws". SIAM journal on numerical analysis, 21(5), pp. 995-1011. 